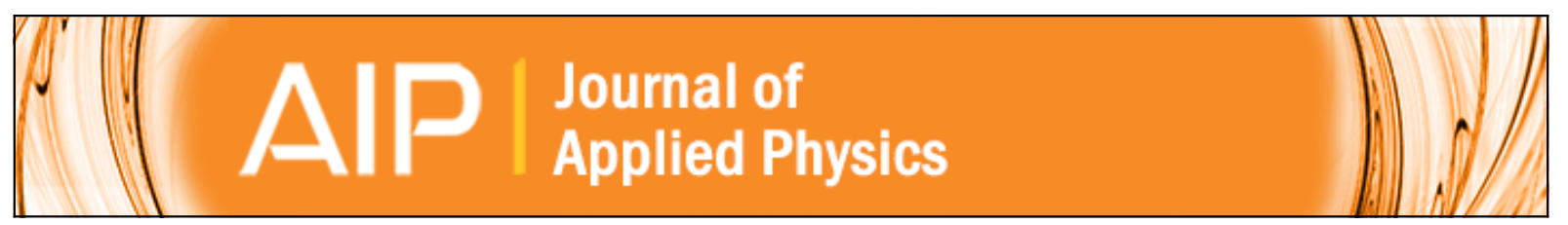

\title{
Multiple-receptor wireless power transfer for magnetic sensors charging on Mars via magnetic resonant coupling
}

Chunhua Liu, K. T. Chau, Zhen Zhang, Chun Qiu, Fei Lin, and T. W. Ching

Citation: Journal of Applied Physics 117, 17 A743 (2015); doi: 10.1063/1.4918624

View online: http://dx.doi.org/10.1063/1.4918624

View Table of Contents: http://scitation.aip.org/content/aip/journal/jap/117/17?ver=pdfcov

Published by the AIP Publishing

\section{Articles you may be interested in}

Erratum: "Experimental demonstration of the equivalence of inductive and strongly coupled magnetic resonance wireless power transfer" [Appl. Phys. Lett. 102, 053904 (2013)]

Appl. Phys. Lett. 107, 049901 (2015); 10.1063/1.4926660

Efficiency analysis of magnetic resonance wireless power transfer with three-dimensional transmitters J. Appl. Phys. 117, 17B516 (2015); 10.1063/1.4914360

Transfer efficiency analysis of magnetic resonance wireless power transfer with intermediate resonant coil J. Appl. Phys. 115, 17A336 (2014); 10.1063/1.4867125

Investigation of energy harvesting for magnetic sensor arrays on Mars by wireless power transmission J. Appl. Phys. 115, 17E702 (2014); 10.1063/1.4854995

Magnetic superlens-enhanced inductive coupling for wireless power transfer J. Appl. Phys. 111, 064902 (2012); 10.1063/1.3692757

\section{AIP $\mid$ APL Photonics}

APL Photonics is pleased to announce Benjamin Eggleton as its Editor-in-Chief

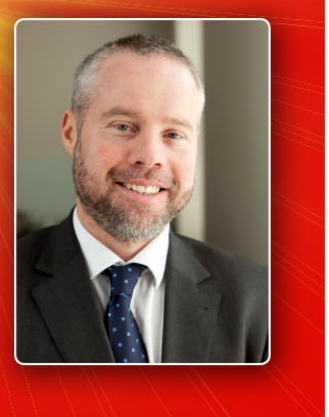




\title{
Multiple-receptor wireless power transfer for magnetic sensors charging on Mars via magnetic resonant coupling
}

\author{
Chunhua Liu, ${ }^{1}{ }^{1, a)}$ K. T. Chau, ${ }^{1}$ Zhen Zhang, ${ }^{1}$ Chun Qiu, ${ }^{1}$ Fei Lin, ${ }^{1}$ and T. W. Ching ${ }^{2}$ \\ ${ }^{1}$ Department of Electrical and Electronic Engineering, University of Hong Kong, Hong Kong, China \\ ${ }^{2}$ Faculty of Science and Technology, University of Macau, Macau, China
}

(Presented 7 November 2014; received 22 September 2014; accepted 11 November 2014; published online 17 April 2015)

\begin{abstract}
This paper proposes a new idea for magnetic sensors charging on Mars, which aims to effectively transmit energy from Mars Rover to distributed magnetic sensors. The key is to utilize wireless power transfer (WPT) to enable multiple receptors extracting energy from the source via magnetic resonant coupling. Namely, the energy transmitter is located on the Mars Rover, whereas the energy receptor is installed in the magnetic sensor. In order to effectively transfer the power, a resonator is installed between the transmitter and the receptors. Based on the proposed idea, the system topology, operation principle, and simulation results are developed. By performing finite element magnetic field analysis, the output power and efficiency of the proposed WPT system are evaluated. It confirms that the Mars Rover carrying with the energy transmitter is capable of loitering around the resonator, while the magnetic sensors on the receptors can be simultaneously charged according to energy-on-demand. (C) 2015 AIP Publishing LLC.

[http://dx.doi.org/10.1063/1.4918624]
\end{abstract}

\section{INTRODUCTION}

In recent years, exploring Mars has attracted much attention: thanks to the successful landing of NASA Spirit Rover, Opportunity Rover, and Curiosity Rover. Since January 25, 2004, the Spirit Rover worked on Mars over 64 months and the Curiosity Rover is still working on Mars. The first two rovers are out of expectation as they were designed for working 3 months only. Hence, it indicates that the electromagnetic devices are capable of adapting to Mars environment. ${ }^{1}$ However, only with several rovers and their limited sensors, it is far from enough to explore Mars.

Reference 1 presents an idea of energy harvesting for magnetic sensors on Mars via wireless power transfer (WPT). In this way, hundreds of magnetic sensors can be located at different areas of Mars for different purposes. The previous idea was to use one transmitter on the Mars Rover wirelessly transferring the power to one magnetic sensor. Thus, at any time, only one magnetic sensor could harness the power from the Mars Rover. Although this idea is quite simple and practical, it takes a long time to sequentially transfer the power to multiple magnetic sensors.

This paper is to propose a new multiple-receptor WPT system, which enables the Mars Rover transmitting energy to charge several magnetic sensors simultaneously. To improve the effectiveness of WPT, the approach of magnetic resonant coupling (MRC) $)^{2,3}$ is adopted for the proposed system. Specifically, a transmitting pad is mounted on Mars Rover, a receiving coil is mounted on each magnetic sensor, and a resonator is located between the Mars Rover and all magnetic sensors. Thus, the Mars Rover carrying with the

\footnotetext{
a) Author to whom correspondence should be addressed. Electronic mail: chualiu@eee.hku.hk.
}

transmitter can loiter around so that the magnetic sensors can be simultaneously charged via the resonator according to the energy-on-demand (EOD). By using finite element magnetic field analysis, the output power and efficiency of the proposed MRC-based multiple-receptor WPT system will be assessed to confirm its validity.

\section{MRC-BASED MULTIPLE-RECEPTOR WPT SYSTEM ON MARS}

Fig. 1 shows the proposed multiple-receptor WPT system with MRC, which mainly includes a Mars Rover, tens of distributed magnetic sensors on Mars surface, and some inbetween resonators. The Mars Rover is equipped with a photovoltaic (PV) panel for solar energy harvesting, a control unit, a battery tank, a WPT transmitter, and other apparatus. The magnetic sensors are allocated on Mars surface for different purposes, which are mounted with a WPT receiving coil, a battery, and a specific sensor. The in-between resonators are located between the Mars Rover and some magnetic sensors so as to improve the effectiveness of energy transmission. Hence, the multiple-receptor WPT system is

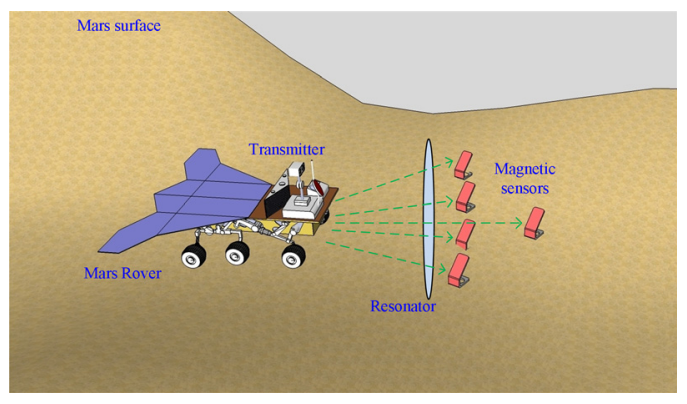

FIG. 1. Proposed multiple-receptor WPT system on Mars. 
designed to enable the Mars Rover charging multiple magnetic sensors.

This multiple-receptor WPT system with MRC for Mars exploration should meet five main criteria: ${ }^{1}$ (1) The Mars Rover has the capability to harness solar energy via its PV panel and store the energy in its battery tank; (2) the magnetic sensors and in-between resonators can be set up at different places on Mars surface for specific purposes; (3) when the magnetic sensors send a request for EOD, the Mars Rover runs around them and performs wireless charging of these sensors; (4) the transmitter, resonator, and magnetic sensor should be designed with proper sizes; for instance, their diameters are normally limited to $500 \mathrm{~mm}, 400 \mathrm{~mm}$, and $300 \mathrm{~mm}$, respectively; and (5) the charging distance from the Mars Rover to magnetic sensors is around $500-1000 \mathrm{~mm}$ to ensure effective and efficient power transfer.

Fig. 2 shows the circuit topology of the proposed multiple-receptor WPT, which mainly consists of three parts, namely, the transmitting coil, resonant coil, and receiving coils with loads. The operating principle is as follow. First, the energy source with the transmitting coil operates at its resonant frequency. Meanwhile, the resonant coil operates at the same frequency, which serves an energy repeater. ${ }^{4,5}$ Finally, the receiving coils with loads are able to extract the energy simultaneously. It is worthy to mention that the farthest receiving coil can extract more energy than the other four receiving coils because those four nearer coils also play the role of repeater for the farthest receiving coil.

It should be noted that, at any time, the five receiving coils need to operate at the same resonant frequency to enable the targeted charging. Meanwhile, the Mars Rover can charge another set of magnetic sensors with another resonant frequency. Therefore, for the proposed multiple-receptor WPT system, the Mars Rover loiters around the magnetic sensors and then tunes its operating frequency to match with the resonant frequency of the magnetic sensors and the corresponding in-between resonator to effectuate WPT upon EOD. Essentially, the load is the battery of the magnetic sensor.

\section{RESULTS AND DISCUSSION}

Fig. 3 is the geometry of the pads and coil for investigation. The transmitting pad has the outside diameter of $480 \mathrm{~mm}$ in which the transmitting coil is wound from the inside diameter of $180 \mathrm{~mm}$ to the outside diameter of $360 \mathrm{~mm}$ while the spoke radially extends from the inside diameter of $80 \mathrm{~mm}$ to the outside diameter of $420 \mathrm{~mm}$ so as to strengthen the magnetic flux for transmission with the outside and inside diameter of $420 \mathrm{~mm}$ and $80 \mathrm{~mm}$. The resonant coil is the same as the transmitting coil, whereas the receiving coil has the inside

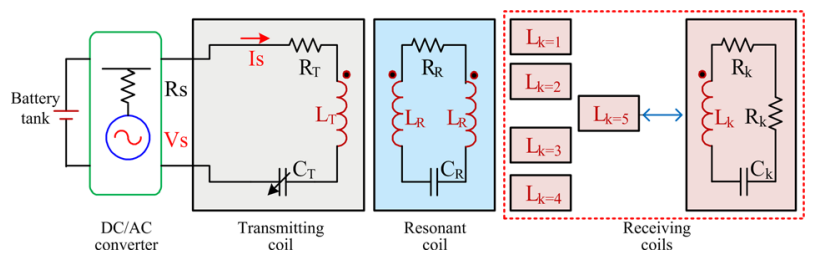

FIG. 2. Circuit topology of proposed WPT system for Mars Rover application.

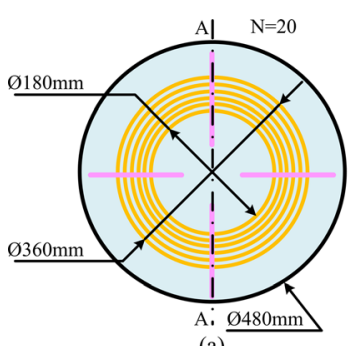

(a)

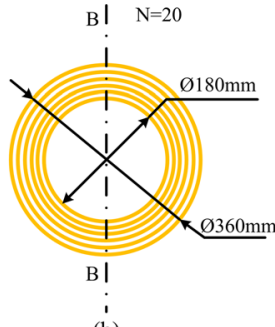

(b)

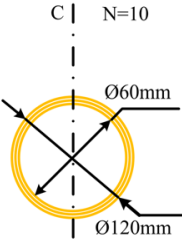

C I

(c)

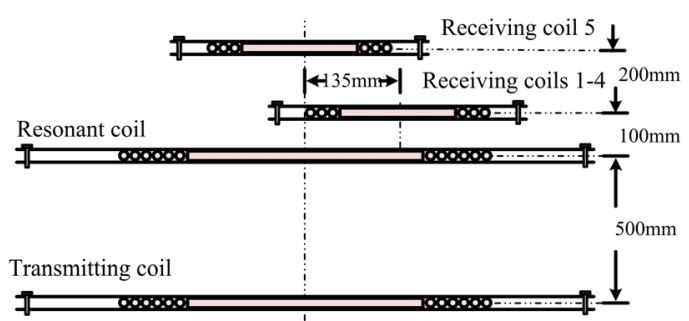

(d)

FIG. 3. Geometry of pads. (a) Transmitting coil. (b) Resonant coil. (c) Receiving coils. (d) Displacements among coils.

and outside diameters of $60 \mathrm{~mm}$ and $120 \mathrm{~mm}$, respectively. The displacements among the coils are listed in Fig. 3. The first four receptors are located with a distance of $600 \mathrm{~mm}$ from the Mars Rover, while the fifth receptor is $800 \mathrm{~mm}$ away. By using finite element magnetic field analysis, namely, the JMAG software tool, ${ }^{6}$ the performance of the proposed system can be thoroughly analyzed.

First, the magnetic field distribution of the proposed system with the resonant frequency of $5 \mathrm{MHz}$ and input current of $5 \mathrm{~A}$ is simulated as depicted in Fig. 4. It can be seen that the flux lines go through the five receiving coils from the transmitting coil via the resonant coil. Also, the fifth receiving coil attracts most of flux lines from the first four receiving coils. It means that the fifth receiving coil is able to harness most of the transmitted energy from the Mars Rover. Moreover, it indicates that the proposed WPT system is

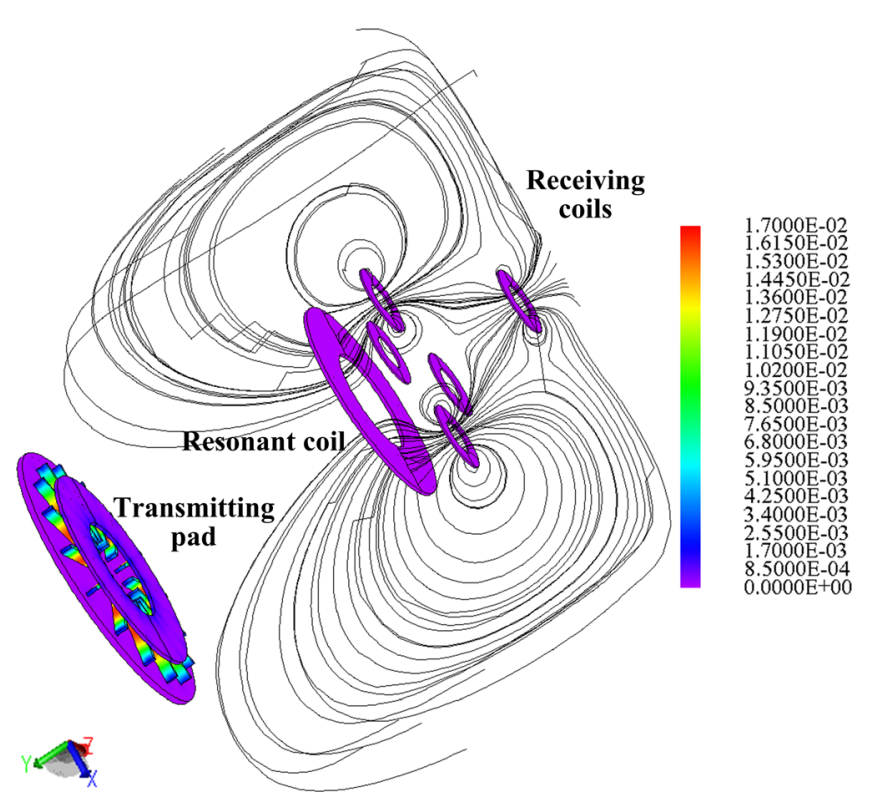

FIG. 4. Magnetic field distribution with resonant frequency of $5 \mathrm{MHz}$ and input current of $5 \mathrm{~A}$. 


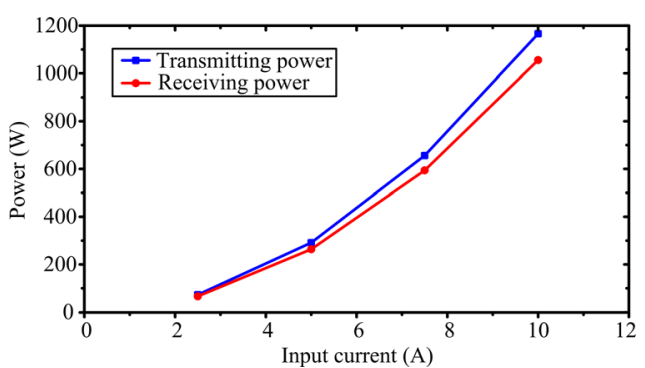

FIG. 5. Total power with resonant frequency of $5 \mathrm{MHz}$ under different input currents.

capable of transmitting energy from the Mars Rover to multiple magnetic sensors via the resonant coil.

Second, the power performance of the proposed multiplereceptor WPT system is calculated and shown in Fig. 5, which operates at the resonant frequency of $5 \mathrm{MHz}$ under different input currents. It can be observed that the total receiving power is from $66 \mathrm{~W}$ to $1055.5 \mathrm{~W}$ when the input current is applied from $2.5 \mathrm{~A}$ to $10 \mathrm{~A}$. As expected, the receiving power increases with the increment of input current. Also, the efficiency keeps nearly the same at $90.6 \%$, which can be observed from both power curves. Furthermore, it indicates that with the resonant frequency up to the megahertz range, the Mars Rover is able to efficiently transfer its energy to different magnetic sensors at the desired power value.

In addition, Fig. 6 shows the power waveforms of the proposed system under different input currents when the Mars Rover increases the resonant frequency to $7.5 \mathrm{MHz}$ in response to the demand from magnetic sensors. It can be seen that the receiving power increases significantly, namely, from $131.8 \mathrm{~W}$ to $2108.8 \mathrm{~W}$ when the input current is varied from $2.5 \mathrm{~A}$ to $10 \mathrm{~A}$. The corresponding efficiency also keeps nearly the same at $90.8 \%$. Thus, it illustrates that the receiving power has a significant improvement when adopting a higher resonant frequency. Also, it indicates that when those magnetic sensors utilize a higher resonant frequency, they have a better capability of energy extraction.

Finally, comparative performances of the system with the resonant frequencies of $5 \mathrm{MHz}$ and $7.5 \mathrm{MHz}$ are analyzed. Fig. 7 gives the comparison of receiving powers under different input currents. It can be found that under the same resonant frequency, if the input current is increased by $100 \%$, the receiving power will increase over $200 \%$. Moreover, it shows that if the resonant frequency is

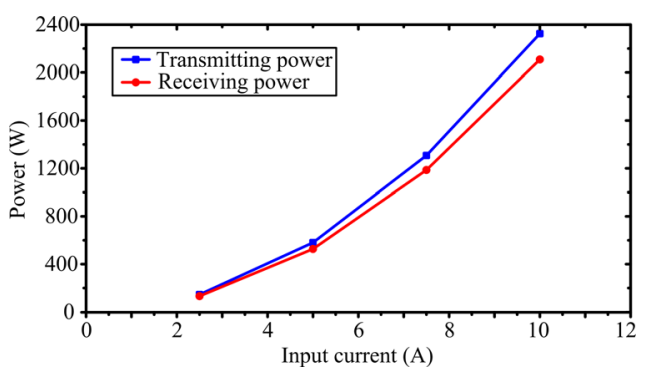

FIG. 6. Total power with resonant frequency of $7.5 \mathrm{MHz}$ under different input currents.

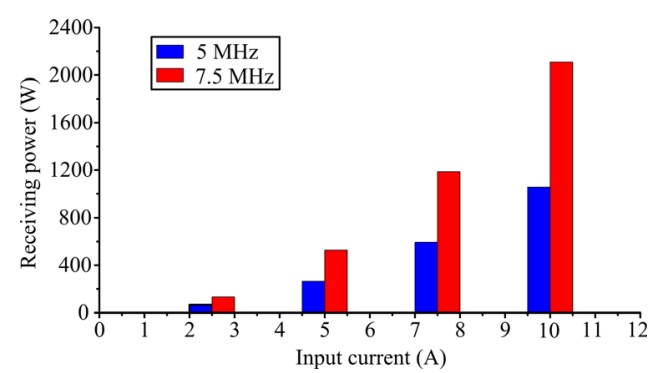

FIG. 7. Comparison of receiving powers with resonant frequencies of $5 \mathrm{MHz}$ and $7.5 \mathrm{MHz}$ under different input currents.

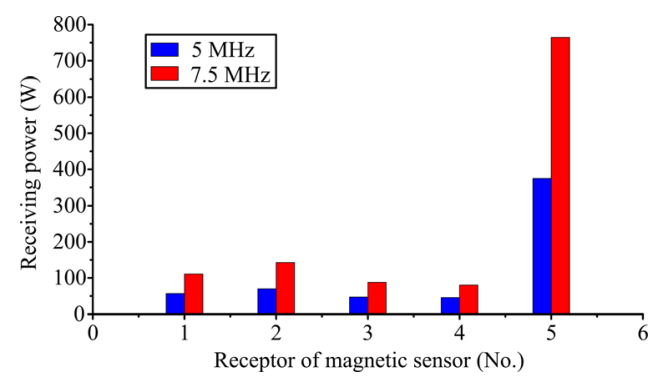

FIG. 8. Comparison of receiving powers of five receptors with resonant frequencies of $5 \mathrm{MHz}$ and $7.5 \mathrm{MHz}$ under input current of 7.5 A.

increased by $50 \%$, namely, from $5 \mathrm{MHz}$ to $7.5 \mathrm{MHz}$, the receiving power will increase by about $100 \%$ under the same input current.

Moreover, Fig. 8 shows the comparison of receiving powers of the five receptors. It can be found that the fifth receptor can extract much higher energy than the other four receptors, namely, about one half of the total receiving power of all receptors. It verifies that under the same resonant frequency, the first four receiving coils play the role of repeater for the fifth receiving coil. In addition, Fig. 8 also shows that the receiving powers of the first four receptors have no big difference because they are distributed with the same distance from the transmitter.

Therefore, the proposed multiple-receptor WPT system can efficiently transmit energy from the Mars Rover to different magnetic sensors via the in-between resonator. Also, the results confirm that this system is able to transfer the energy with the distance from $500 \mathrm{~mm}$ up to $1000 \mathrm{~mm}$ with high power levels.

\section{CONCLUSIONS}

In this paper, a new multiple-receptor WPT system with MRC is presented for Mars exploration. The idea is accomplished and verified. The main contributions are summarized as follows: (1) The whole system is simple and practical, which can simultaneously charge multiple magnetic sensors by using the Mars Rover; (2) the charging power and efficiency are sufficient for the distance from $500 \mathrm{~mm}$ and up to $1000 \mathrm{~mm}$; (3) the resonant frequency should be set at the megahertz range and the charging current should be set at several amperes; and (4) tens or even hundreds of magnetic sensors are suggested to locate at different areas on Mars for different purposes. 


\section{ACKNOWLEDGMENTS}

This work was supported and funded by a research grant (Project Code: SPF 201409176208) from the University of Hong Kong in Hong Kong Special Administrative Region, China.

${ }^{1}$ C. Liu, K. T. Chau, C. Qiu, and F. Lin, J. Appl. Phys. 115, 17E702 (2014).
${ }^{2}$ A. Kurs, A. Karalis, R. Moffatt, J. D. Joannopoulos, P. Fisher, and M. Soljacic, Science 317, 83 (2007).

${ }^{3}$ A. P. Sample, B. H. Waters, S. T. Wisdom, and J. R. Smith, Proc. IEEE 101(6), 1343 (2013).

${ }^{4}$ I. Sasada, J. Appl. Phys. 111, $07 E 733$ (2012).

${ }^{5}$ S. Cheon, Y.-H. Kim, S.-Y. Kang, M. L. Lee, J.-M. Lee, and T. Zyung, IEEE. Trans. Ind. Electron. 58(7), 2906 (2011).

${ }^{6}$ C. Liu, K. T. Chau, and J. Z. Jiang, IEEE. Trans. Ind. Electron. 57(12), 4055 (2010). 